\title{
A Fast BPNN Based Image Deblurring Method
}

\author{
Ren Xiaozhi \\ College of Mechanical Engineering, Guangxi \\ University, xiaozhiren826@163.com, Nanning, \\ Guanxi , 530004
}

\author{
Bin binchao \\ Tobacco Monopolgy Rureau of Guangxi Zhuang \\ Autonomous Region(Guangxi Zhuang Autonomous Region \\ Branch of CNTC), Nanning, Guanxi 530022
}

\begin{abstract}
This paper proposes a fast deblurring method based on back propagation neural networks (BPNN), in which the symmetry of blur function is utilized to reduce the size of network. In the image block used to be training vector, the pixels that have the same distance with centrral pixel are set the same weight in BPNN. This set of weight is realized by adding all the pxiels that have the same distance to be one input data of BPNN. Comparing with the classic BPNN based deblurring method in which the symmetry of blur function is not considered, the proposed method decreased the computation consumption largely. At the same time, the performance of proposed method is improved. Several experiments results testify the superiority of proposed deblurring method.
\end{abstract}

Keywords-image deblurring; back propagation neural networks; blur function.

\section{INTRODUCTION}

Image degradation is hard to avoid in imaging system because of many advent reasons such as atmospheric turbulence, camera movement during the exposure progress, out-of-focus etc. Image restoration is concerned as reconstruction of the original image from degraded image, which is one of important topics in image processing and computer vision. Image blurring is the most common type of degradation in imaging process. The linear shift invariant degradation model can be expressed as

$$
g(m, n)=h(m, n) * f(m, n)+n(m, n)
$$

Where $g, h$ and $f$ are the blurred image, the point-spread function (PSF) of the blurring system and the original image respectively, $n_{\text {is additional }}$ noise, * is convolution operator.

In order to restore the original image $f(m, n)$ from the degraded image $g(m, n)$, deblurring is usually required. Over the past several decades, the research of image restoration gains much attention. The simplest method is inverse filter in frequency domain. But the inverse filter is very sensitive to numerical value in practical implement. Wiener filter is another deblurring method [1]. Wiener filter prone to producing ringing phenomenon and filtering performance has certain restrictions. The iterative methods such as the Richardson-Lucy method [2, 3] and the Landweber method [4] are also common deblurring methods.

With the wide application of neural networks, there have been many works on image restoration using neural networks [5-10]. In literature [5-7], the back propagation neural networks (BPNN) is used to perform image restoration by supervise learning. The hopfield networks is also used to resolve the cost function of image restoration [8-10].

In this paper, we proposed a fast BPNN based deblurring method by considering the symmetry of blur function. In many circumstance, the blur function is symmetry such as Gaussian blur function and defocus blur function. The computation speed is largely reduced because of the size of BPNN is smaller than the classic BPNN based deblurring method. At the same time, the constraint of the symmetry of blur function is added to the method, which will improve the deblurring performance and increase the robustness.

The rest of the paper is organized as follows. Section 2 outlines the proposed image deblurring method based on BPNN. Section 3 describes the organization of training data and BPNN structure used in the proposed fast deblurring method. Experimental results are presented in section 4. Finally, the conclusions are given in section 5 .

\section{THE IMAGE DEBLURRING METHOD BASED ON BPNN}

The BPNN that is composed of many hierarchical linear mapping units has great non-linear mapping capability. It can approximate any non-linear mapping function theoretically. We assume that the blur is shift invariant and the blur function is symmetry, which is in according with fact in many conditions.

The fundamental idea is selecting a pair of images which are blurred image and original definitional image separately. This pair of images is used as training samples. By training, the BPNN can learn complex mapping relationship between blurred image and definitional image. The trained BPNN will have learned the degradation process. We can get definitional image by input other blurred images into the trained BPNN. 
We cannot directly implement above training process as the expected output image is unknown. A common method is to select some representative images without blurring effects as expectation output samples images, then performing degradation process to get blurred images as input samples. This method requires that the texture of sample images be similar to the images needed to be deblurred; otherwise we may not get satisfactory results as BPNN has not infinite generalization ability.

We use another method in this paper. The blurred observation image is degraded to get more blurred image. The more blurred image and blurred observation image are regarded as train samples of BPNN. Deblurred image will be got by input original blurred observation images into this trained BPNN. Because we use the images itself as training samples, there will have not the problem of generalization ability. The flow chart of the proposed algorithm is shown in figure 1.

\section{THE ORGANIZATION OF TRAINING DATA AND BPNN STRUCTURE}

The training data of input and output samples should be organized suitably. The simplest method is that all pixels from input sample image are used as input of BPNN and all pixels of output sample image are used as output of BPNN. This method need huge amount of neural neurons and storage capacity, which will not be feasible if image size is big.

From the imaging model in equation (1), we know that each blurred pixel is produced by several pixels of definitional image around it. Oppositely, each pixel of definitional image is related only with several pixels in blurred image around it. In this paper, the $5 \times 5$ image block is taken as input training vector of BPNN and the central pixel is output training vector. The $5 \times 5$ image block is shifted over the total image. If the image has $m \times n$ size, there will have $(m-4) \times(n-4)$ training vectors.

In the classic BPNN based deblurring method, all pixels of $5 \times 5$ image block have different weight values in BPNN. Figure 2 (a) shows this organization manner. As this method does not consider the symmetry of blur function, which will be prone to over fitting. In figure 2(b) we propose another effective data organization and mapping method. The surrounding pixels around the central pixel that have the same distance should have the same weight values of BPNN. Obviously, this mapping method will largely reduce the input data. Simultaneously it also means that the constraint condition of symmetry of blur function has added to the BPNN. The phenomenon of over fitting will be reduced and the robustness will be increased comparing with the classic data organization method.
The number of input and output pixels of training samples determines the number of nodes of the input and output layer of BPNN, therefore in the classic BPNN based deblurring method, the input and output of BPNN should have 25 nodes and 1 node respectively. The number of neurons in the hidden layer is the same as that in the input layer. A larger number of neurons in the hidden layer do not contribute to an improved performance. The network is composed of three layers and the neurons of every layer are 25, 25 and 1 which correspond to the input 、hidden and output layer respectively.

According to the proposed data organization method as shown in figure 2(b), the input and output of BPNN should only have 6 nodes and 1 node respectively. The hidden layer has also 6 nodes, which has the same nodes number with input nodes. Figure 3 presents the BPNN architecture used in the proposed deblurring method. The pixels that have the same distance with the central pixel are added and the sum is considered as one input data.

\section{EXPERIMENTAL RESULTS}

In the simulations, we used MATLAB Neural Network ToolBox to implement back-propagation Neural Network. The gray-scale images with $128 \times 128$ pixels are tested. For uniformity, all pixel intensity values are normalized to $[0,1]$. We use a computer with Pentium CPU of $2.80 \mathrm{GHz}$ to perform the experiments.

In the first experiment, a satellite image is blurred by Gaussian kernel whose support domain is $5 \times 5$ and standard deviation is 1 . During training, each pixel and its twenty-four surrounding neighbors in the $5 \times 5$ region are presented as $25 \times 1$ input vector to the classic BPNN based learning system and $6 \times 1$ input vector to the proposed BPNN based learning system. The output value for each input vector is obtained from the intensity value in the original image corresponding to the location of the center pixel in the $5 \times 5$ image block. The experiment results are shown in figure 4. As can be seen, the results of proposed fast method have higher definition than the result of classic method. We use PSNR (dB) and computation time (s) as quantitative indices to compare the two BPNN based deblurring method. The values of PSNR and computation time are shown in table 1 . Comparing with the classic BPNN based deblurring method, the PSNR of proposed method is improved about $1 \mathrm{~dB}$ and the computation time is about onethird of classic method.

Next we show another results corresponding to badly blurred image, which are shown in figure 5 . In the experiment, another satellite image is badly blurred by Gaussian kernel whose support domain is $5 \times 5$ and standard deviation is 2 . From (c) and (d), 
the two deblurred result are similar. The PSNR and compution time are given in table 2. The PSNR values of (c) and (d) are similar. The computation time of proposed method is also about one-third of classic method.

\section{CONCLUSIONS}

A fast BPNN based deblurring scheme is developed in this paper. The basic idea behind the proposed approach is that the blur function is symmetry in many cases such as Gaussian blur function 、 defocus blur function and so on. This symmetry of blur function is then utilized to speed up the training and computation of BPNN. Three experiments shows that the computation time of proposed method is about one-third of classic BPNN based deblurring method and the PSNR increased about $1 \mathrm{~dB}$ comparing with the classic method.

\section{REFERENCES}

[1] Rafael C. Gonzalez, and Richard E. Woods, Digital Image Processing[M]. Addison-Wesley Longman Publishing Co., Inc., Boston, MA, (1992)

[2] W. H. Richardson, Bayesian-based iterative method of image restoration[J], J. Opt. Soc. Amer., 62, 55-59, (1972).
[3] L. B. Lucy, An iterative technique for the rectification of observed distributions[J], Astronom. J., vol. 79, (1974).

[4] Lei Liang and Yuanchang $\mathrm{Xu}$, Adaptive Landweber Method to Deblur Images[J] IEEE SIGNAL PROCESSING LETTERS, 10(5), 129-132 (2003).

[5] J.C. Gillette, T.M. Stadtmiller, and R.C. Hardie, Aliasing reduction in staring infrared imagers utilizing subpixel techniques[J], Opt. Eng., 34(11), 3130-3137 (1995).

[6] K. Sivakumar, and U.B. Desai, Image-restoration using a multilayer perceptron with a multilevel sigmoidal function[J], IEEE transactions on signal processing, 41(5), 2018-2022 (1993).

[7] Perry, S.W.Varjavandi, and P.Ling Guan, Adaptive image restoration using a perception based error measurement[C],in Proceedings of Electrical and Computer Engineering, pp.1585-1588 (2004).

[8] Ya-Dong Wu, Qing-Xin Zhu, Shi-Xin Sun, and Hong-Ying Zhang, Image restoration using variational PDE-based neural network[J], Neurocomputing, 69, 2364-2368 (2006).

[9] Zhou Y T, Chellappa R, and Jenkins B K, Image restoration using a neural network,[J] IEEE Trans Acoust Speech Signal Processing, 36(7),1141-1151(1988).

[10] Paik J K, and Katsaggelos A K, Image restoration using a modified Hopfield network[J] IEEE Trans Image Processing, 1(1), 49-63(1992).

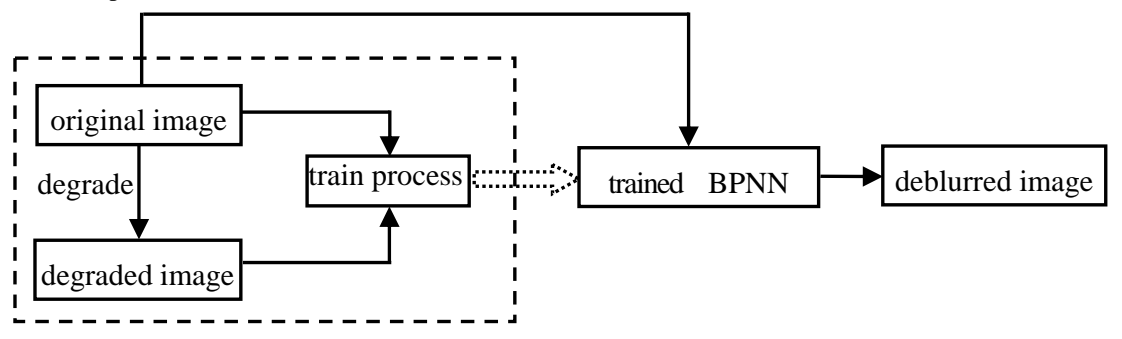

Figure 1. The flow chart of the proposed algorithm.

(a)

\begin{tabular}{|c|c|c|c|c|}
\hline 1 & 2 & 3 & 4 & 5 \\
\hline 6 & 7 & 8 & 9 & 10 \\
\hline 11 & 12 & 13 & 14 & 15 \\
\hline 16 & 17 & 18 & 19 & 20 \\
\hline 21 & 22 & 23 & 24 & 25 \\
\hline
\end{tabular}

(b)

\begin{tabular}{|l|l|l|l|l|}
\hline 6 & 5 & 4 & 5 & 6 \\
\hline 5 & 3 & 2 & 3 & 5 \\
\hline 4 & 2 & 1 & 2 & 4 \\
\hline 5 & 3 & 2 & 3 & 5 \\
\hline 6 & 5 & 4 & 5 & 6 \\
\hline
\end{tabular}

Figure 2. The relations between input and output training vector of classic method (a) and proposed method (b). The center black pixel is output pixel and the total 25 pixels are input vector.

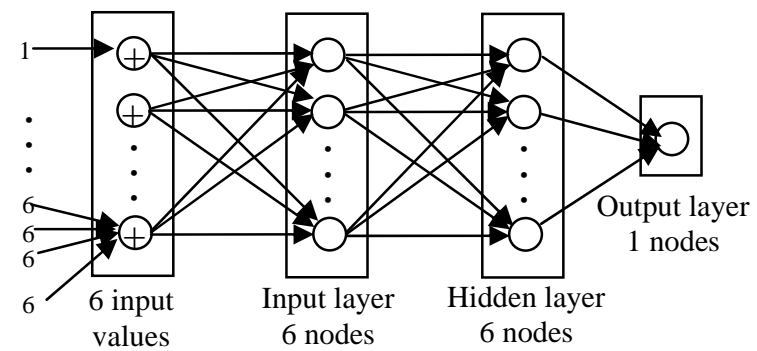

Figure 3. The BPNN architecture used in the proposed deblurring method. 

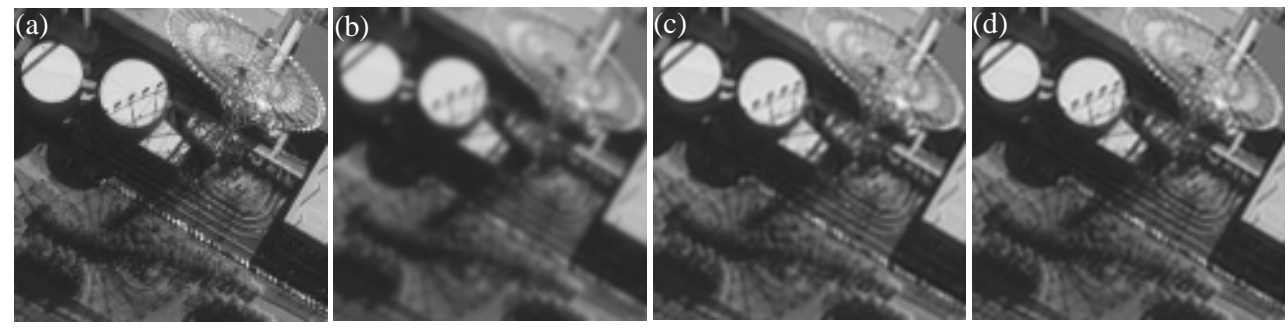

Figure 4. The first test results. (a) is original image; (b) is blurred image with standard deviation 1; (c) is the deblurred image by the classic method based BPNN; (d) is the deblurred image by proposed fast method.
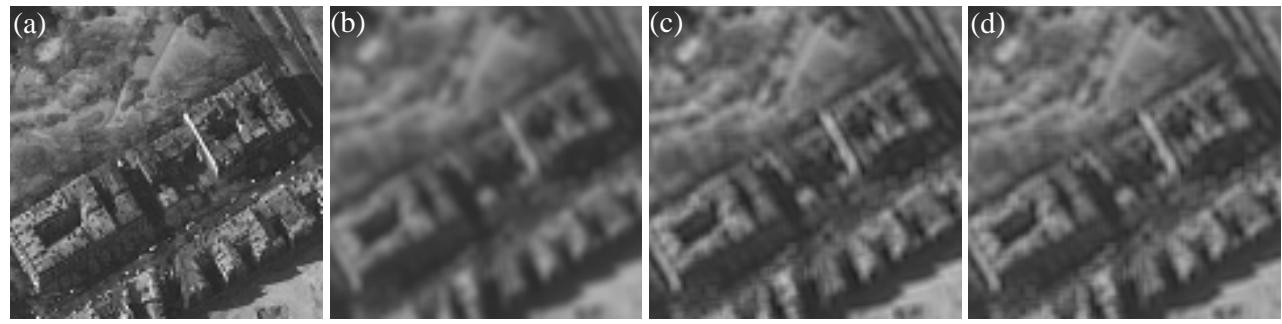

Figure 5. The second test results. (a) is original image; (b) is blurred image with standard deviation 2; (c) is the deblurred image by the classic method based BPNN; (d) is the deblurred image by proposed fast method.

TABLE I. THE COMPARISON OF ALGORITHM PERFORMANCE TO LITTLE BLURRED IMAGE.

\begin{tabular}{cccc}
\hline image & Blurred image & Classic method & Proposed method \\
\hline PSNR (dB) & 25.9143 & 29.4709 & 30.3266 \\
Computation time(s) & & 88.1735 & 21.6871 \\
\hline
\end{tabular}

TABLE II. THE COMPARISON OF ALGORITHM PERFORMANCE TO BADLY BLURRED IMAGE.

\begin{tabular}{cccc}
\hline image & Blurred image & Classic method & Proposed method \\
\hline PSNR (dB) & 22.4830 & 25.6119 & 25.9784 \\
Computation time(s) & & 129.7731 & 30.8505 \\
\hline
\end{tabular}

